\title{
A Systematic Review of the Economic and Humanistic Burden of Illness in Irritable Bowel Syndrome and Chronic Constipation
}

\author{
Dave Nellesen, PhD, MBA; Kimberly Yee, MPH; Anita Chawla, PhD; \\ Barbara Edelman Lewis, PhD, MHA; and Robyn T. Carson, MPH
}

\begin{abstract}
BACKGROUND: Irritable bowel syndrome (IBS) and chronic constipation (CC) are common functional gastrointestinal disorders affecting $14 \%$ and $20 \%$ of the U.S. population, respectively. Reviews of the evidence on the burden of illness associated with IBS and CC have not been comprehensive in scope and have not provided an assessment of the distribution of health care costs across categories of resource use.
\end{abstract}

OBJECTIVES: To (a) identify studies from any geographic region or country perspective that measure the economic burden of the disease; (b) analyze the direct (medical, drug, and other components) and indirect costs of illness; and (c) assess published evidence of the humanistic burden as measured by quality of life (QOL).

METHODS: An electronic literature search was conducted using journal databases, including MEDLINE, The Cochrane Library, EconLit, CINAHL, and Digestive Disease Week meeting abstracts. Specific search terms used were "irritable bowel syndrome" and "chronic constipation." In databases that accommodated Boolean searches, terms related to economic and quality of life outcomes were incorporated. Studies were included if they evaluated patients with an IBS or CC diagnosis and quantitatively measured the economic or humanistic burden of disease. Results were descriptively analyzed.

RESULTS: The search identified a total of 882 unique publications. Thirtyfive articles and abstracts met the inclusion criteria. Studies included 1,706 IBS-C, 2,264 IBS-D, 2,892 IBS-A, 15,830 IBS unclassified, and 1,278 CC patients. Nineteen of 35 studies assessed cost-of-illness endpoints, and from the U.S. perspective, the direct cost per-patient for IBS ranged from $\$ 1,562$ to $\$ 7,547$ per year, while direct costs of CC ranged from $\$ 1,912$ to $\$ 7,522$ per year. From the U.S. perspective, the indirect costs of IBS ranged from $\$ 791$ to $\$ 7,737$ per year, and no study assessed the indirect costs of CC. For IBS, data on the distribution of costs attributable to categories of resource use varied widely, particularly outpatient costs $(12.7 \%$ to $>50 \%$ of total costs), inpatient costs $(6.2 \%$ to $40.8 \%)$, and pharmacy or drug costs $(5.9 \%$ to $46.6 \%)$. Comparable data on CC were not identified. Nineteen studies of IBS patients measured the humanistic burden of disease; 14 studies utilized SF-36; and within-study domain scores were significantly lower in IBS patients compared with non-IBS controls. Only 1 study of CC patients reported humanistic burden of disease.

CONCLUSIONS: The studies identified in the systematic review varied in the method used to identify patients with IBS and CC. Results were not typically reported by IBS subtype. We observed a large variation in attributable direct and indirect costs and drivers of these costs. Future research should refine burden of illness estimates to subtypes so that estimates associated with IBS-C and CC are differentiated.

J Manag Care Pharm. 2013;19(9):755-64

Copyright $\odot 2013$, Academy of Managed Care Pharmacy. All rights reserved.

\section{What is already known about this subject}

- Irritable bowel syndrome (IBS) and chronic constipation (CC) are 2 of the most common functional gastrointestinal disorders estimated to affect nearly 1 in 5 North Americans

- It has been estimated that the annual cost of IBS in the United States is between $\$ 1.7$ and $\$ 10$ billion in direct medical costs, excluding prescription and over-the-counter drugs, and up to $\$ 20$ billion in indirect costs.

- Diagnostic criteria for IBS and CC have changed over time and have been applied inconsistently, making the investigation of the burden of illness challenging.

\section{What this study adds}

- This systematic assessment of the economic and humanistic burden of illness identified numerous studies documenting burden of illness measured in economic and humanistic terms.

- Direct costs of IBS ranged from $\$ 1,562$ to $\$ 7,547$ per year, and the indirect costs ranged from $\$ 791$ to $\$ 7,737$ per year, while the direct costs of CC ranged from $\$ 1,912$ to $\$ 7,522$ per year. Indirect costs for CC were not identified in this systematic review.

- The distribution health care cost of IBS varied widely, particularly outpatient costs $(12.7 \%$ to $>50 \%$ of total costs), inpatient costs (6.2\% to $40.8 \%$ ), and pharmacy or drug costs (5.9\% to $46.6 \%$ ). Only 2 studies reported health care cost of patients with CC. Neither study reported cost by standard categories of service.

- In studies that compared IBS with a non-IBS control population, quality of life (as measured by SF-36) was significantly lower in IBS patients than in controls. Only 1 study of CC patients reported humanistic burden.

- Published estimates have yet to address burden of illness according to disease subtypes.

I rritable bowel syndrome (IBS) and chronic constipation (CC) are common functional gastrointestinal disorders (FGIDs) characterized by abnormalities in motility and visceral hypersensitivity. ${ }^{1}$ IBS is defined by the presence of symptoms of abdominal pain or discomfort associated with disturbed defecation, ${ }^{2}$ while chronic or functional constipation is characterized by infrequent bowel movements (BM), hard or lumpy stools, straining, and a sensation of incomplete rectal evacuation. ${ }^{3}$ IBS is one of the most common FGIDs, ${ }^{4}$ with an 
estimated prevalence of $14.1 \%$ in a survey of U.S. patients. ${ }^{5}$ IBS affects both men and women, but approximately $60 \%$ to $75 \%$ of sufferers are women. ${ }^{6,7} \mathrm{CC}$ is estimated to affect up to $20 \%$ of the North American population. ${ }^{8}$ Although women report CC approximately twice as often as men, ${ }^{9}$ in U.S.-based surveys, CC occurred only moderately more frequently in women, at rates of $56 \%{ }^{10}$ and $62 \% .^{11}$ In both men and women, the incidence of CC increases with age. ${ }^{11}$

IBS includes several clinically distinct different subtypes. The Rome III criteria ${ }^{1}$ distinguish IBS subtypes by predominant bowel habit disturbance: IBS with constipation (IBS-C), IBS with diarrhea (IBS-D), and mixed or alternating bowel pattern with both diarrhea and constipation (IBS-M or IBS-A). Reports on the relative proportions of IBS subtypes range from $5.2 \%$ to $66 \%$ for IBS-C, $0.8 \%$ to $33.9 \%$ for IBS-D, and $5.2 \%$ to $33.1 \%$ for IBS-A. ${ }^{12}$ The chronic, episodic nature of IBS and CC and the limited sensitivity and specificity in diagnosis, as well as changes in diagnostic criteria over time, present substantial challenges for clinicians and researchers in characterizing the spectrum of patients' experiences.

A number of studies have sought to measure economic and humanistic burden of illness attributable to IBS and CC. One systematic review published in 2002 assessed the healthrelated quality of life (HRQOL) burden of IBS, ${ }^{13}$ and a review published in 2003 documented the costs of IBS symptom management, direct costs, and productivity costs..$^{14}$ Another review published in 2004 highlighted the considerable economic burden imposed by IBS, with direct medical costs (excluding prescription and over-the-counter drug costs) ranging between $\$ 1.7$ billion and $\$ 10$ billion and indirect costs reaching $\$ 20$ billion..$^{15}$ There have been some studies comparing CC treatments, but no systematic reviews or meta-analyses of the burden of CC have been published. Together, these reviews demonstrate the limited scope of recent research undertaken to understand these conditions, in particular studies that have investigated the burden of illness in specific IBS subtypes.

\section{Objectives}

The main objectives of this systematic review are as follows: to (a) identify studies from any geographic region or country perspective that measure the economic burden of IBS and CC; (b) analyze the direct (medical, drug, and other components) and indirect costs of illness; and (c) assess published evidence of the humanistic burden of disease as measured by effects on HRQOL in IBS and CC. In particular, the goals were to identify studies that specifically assess the burden of disease in patients with CC or the IBS subtypes IBS-C, IBS-D, and IBS-M or IBS$\mathrm{A}$, and to describe aspects of the economic or humanistic burden of disease in patients with CC or an IBS subtype that could provide a better understanding of the disorders, their manifestations, and potential implications in managed care settings.

\section{Methods}

\section{Literature Search}

An electronic literature search was conducted in MEDLINE, MEDLINE In-Process, The Cochrane Library, EconLit, the Cumulative Index to Nursing and Allied Health Literature (CINAHL), and Digestive Disease Week (DDW) meeting abstracts. There were no date restrictions; however, DDW abstracts were only available for the years 2008-2010. Across all databases searched, common keywords included "irritable bowel syndrome" and "chronic constipation." In addition, other terms may have been used to supplement these keywords, depending on the search capabilities and interface of the database. In databases that accommodated Boolean searches, terms specifically related to economic and quality of life outcomes were added. The MEDLINE search limited results to English language articles. All searches were performed in November 2010.

\section{Study Selection Criteria}

Inclusion criteria were (a) patients were specifically described as having an IBS or CC diagnosis, and (b) study quantifiably measured the economic or quality of life (QOL) burden attributable to IBS or CC. Studies were excluded if they assessed the economic or QOL impact of a particular treatment for IBS or CC (studies where impact may be attributable to an intervention rather than to the disease). Case studies and meta-analyses were excluded to minimize the chances of double-counting patients.

\section{Data Abstraction}

Studies were classified according to outcomes (i.e., burden of illness related to economics or QOL or both) and condition of interest (IBS, CC, or both). Where information was available, patient counts were further disaggregated by IBS subtype into IBS-C, IBS-D, and IBS-M or IBS-A. For economic outcomes, quantitative values for direct and indirect costs and narrative information on categories of resource use were collected. For QOL outcomes, overall and domain scores from available QOL scales were collected. Information was also collected regarding study design and patient characteristics. Studies were not evaluated for quality.

\section{Statistical Analyses}

Descriptive analysis was performed for the included studies. Meta-analysis was not performed, since the inclusion criteria were not specific enough to control for variability in study design and patient characteristics.

\section{Results}

\section{Characteristics of the Selected Studies}

The search identified a total of 882 unique publications from all the databases; 35 articles and abstracts met the selection criteria and were included in the data-abstraction step (Figure 1). 


\section{FIGURE 1 Study Selection Schema}

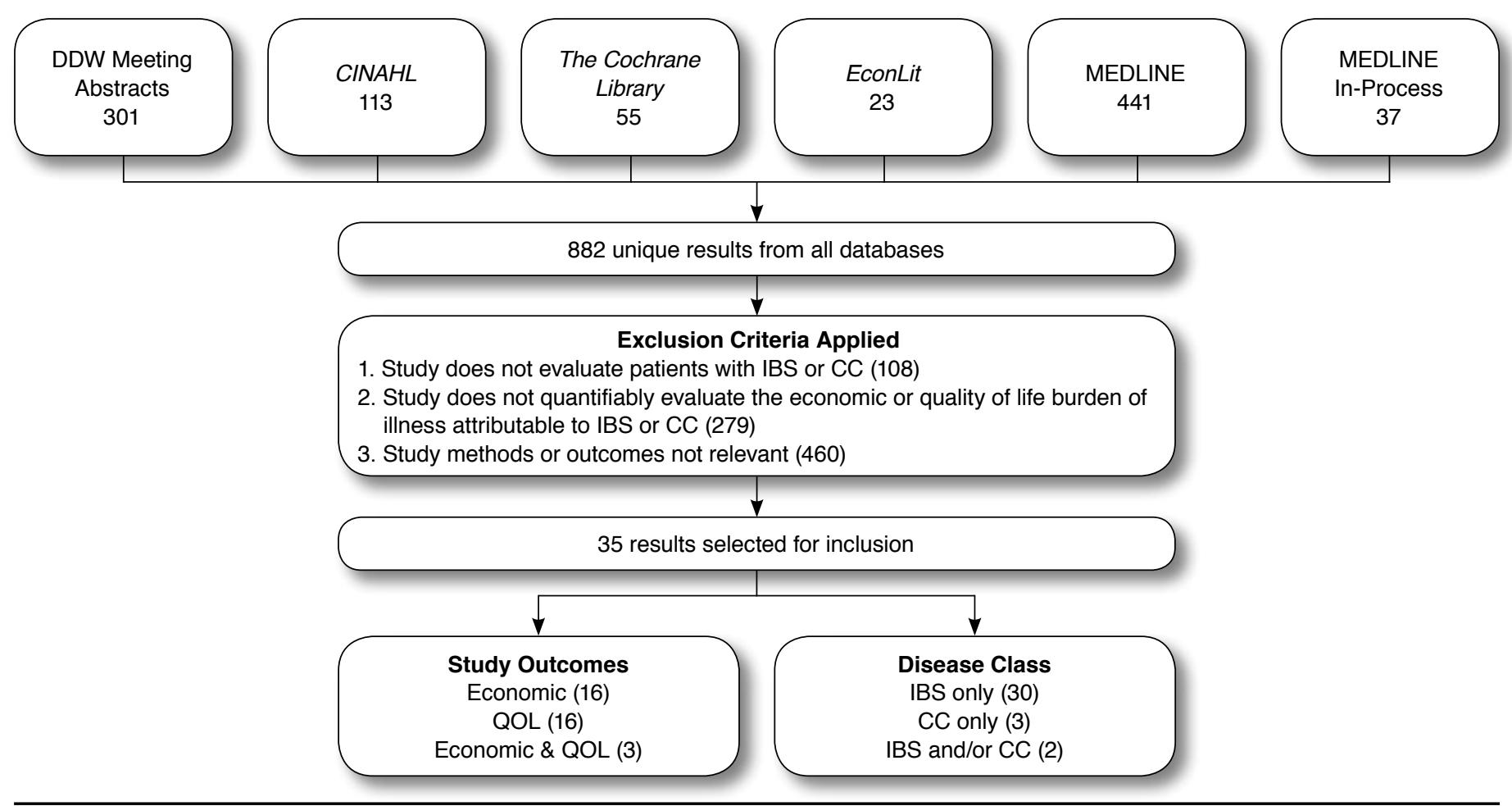

$C C=$ chronic constipation; $C I N A H L=$ Cumulative Index to Nursing and Allied Health Literature; DDW=Digestive Disease Week; IBS =irritable bowel syndrome; QOL= quality of life.

The studies were classified according to study outcomes (economic, humanistic or both) and disease of interest (IBS, CC, or both). Of the 35 studies, 16 evaluated economic burden of disease or humanistic burden of disease only, and 3 assessed economic and humanistic burden of disease. By disease of interest, 30 studies investigated IBS only, 3 studied CC only, and 2 included patients with either IBS or CC. The criteria used for diagnosis of IBS, IBS-C, and CC varied widely (Figure 1). These 35 studies included a total of 63,816 patients with various digestive diseases and related conditions. The IBS and CC population included the following: 1,706 IBS-C, 2,264 IBS-D, 2,892 IBS-A, 15,830 IBS unclassified, and 1,278 CC patients.

The majority of the studies were conducted in the United States $(n=17)$; the remaining studies were conducted in France ( $n=5)$; the United Kingdom ( $n=3)$; Canada, Korea, the Netherlands ( $\mathrm{n}=2$ each); and China, Germany, and Norway or Sweden ( $\mathrm{n}=1$ each). Selected characteristics of the 35 studies are presented in Table 1.

\section{Studies Assessing Cost-of-IIIness Endpoints}

Nineteen of the 35 studies identified reported cost-of-illness outcomes. Eleven studies investigated the direct costs of IBS and/or CC, 1 investigated only the indirect costs, and 7 inves- tigated both the direct and indirect costs. The majority of the cost studies were from the U.S. perspective $(n=12)$; other countries studied were France and United Kingdom ( $\mathrm{n}=2$ each), Canada, Germany, and Norway ( $\mathrm{n}=1$ each). Fifteen of the economic studies were of patients with IBS only; 2 studies were of patients with CC only; and 2 studies included both IBS and CC patients. Only 2 studies specifically examined costs of patients with IBS-C. ${ }^{16,17}$

Categories of resource use and associated direct and indirect costs from these studies are presented in Table 2. From the U.S. perspective, the direct cost per-patient of IBS ranged from $\$ 1,562$ per year (2002 U.S. dollars [USD]; \$2,269 per year in 2012 USD) to $\$ 7,547$ per year (year not reported [NR], published in 2000; \$12,007 per year in 2012 USD). The direct costs of CC ranged from $\$ 1,912$ per year (2002 USD; $\$ 2,778$ per year in 2012 USD) to $\$ 7,522$ per year (2002-2003 USD; $\$ 11,967$ per year in 2012 USD). Direct costs for IBS included those associated with medications, hospitalizations, outpatient services, emergency department visits, and laboratory tests. For IBS, the reported share of costs across categories of health care resource use varied widely, particularly outpatient costs (12.7\% to $>50 \%$ of total costs), inpatient costs (6.2\% to $40.8 \%$ ), and pharmacy or drug costs (5.9\% to $46.6 \%)$. 


\section{TABLE 1 Overview of Selected Studies}

\begin{tabular}{|c|c|c|c|c|c|c|c|c|}
\hline \multirow[b]{2}{*}{ Reference } & \multirow[b]{2}{*}{ Population } & \multirow[b]{2}{*}{ Comparison Population } & \multirow{2}{*}{$\begin{array}{l}\text { Country, } \\
\text { Perspective }\end{array}$} & \multicolumn{5}{|c|}{ Number of Patients } \\
\hline & & & & IBS Total & IBS-C & IBS-D & IBS-A & $\mathrm{CC}$ \\
\hline \multicolumn{9}{|c|}{ Prospective cohort study (IBS only) } \\
\hline Johansson $2010^{30}$ & $\begin{array}{l}\text { Sample of patients with IBS in } \\
\text { Norwegian general practice }\end{array}$ & None & Norway & 164 & & & & \\
\hline \multicolumn{9}{|c|}{ Case-control study (IBS only) } \\
\hline Park 200933 & $\begin{array}{l}\text { Patients aged } 18-75 \text {, symptoms consis- } \\
\text { tent with Rome II criteria, absence of } \\
\text { abnormal colonoscopic exam results }\end{array}$ & $\begin{array}{l}\text { Controls from the general } \\
\text { population }\end{array}$ & Korea & 664 & 168 & 368 & 128 & \\
\hline Faresjö $2007^{34}$ & $\begin{array}{l}\text { Aged 18-65 with first diagnosis of IBS } \\
\text { between 1997-2001 }\end{array}$ & None & Sweden & 347 & & & & \\
\hline Martin $2003^{26}$ & $\begin{array}{l}\text { Patients > } 18 \text { years with ICD-9-CM code } \\
\text { of irritable colon }\end{array}$ & $\begin{array}{l}\text { Control subjects with no } \\
\text { claims for irritable colon, } \\
\text { abdominal pain, or consti- } \\
\text { pation }\end{array}$ & $\begin{array}{l}\text { United } \\
\text { States, } \\
\text { Medicaid }\end{array}$ & 2,546 & & & & \\
\hline Akehurst $2002^{23}$ & $\begin{array}{l}\text { Patients meeting Rome I criteria for IBS } \\
\text { from } 6 \mathrm{GP} \text { practices in the United Kingdom }\end{array}$ & $\begin{array}{l}\text { Controls matched for age, } \\
\text { sex, social characteristics }\end{array}$ & $\begin{array}{l}\text { United } \\
\text { Kingdom }\end{array}$ & 161 & & & & \\
\hline Patel $2002^{28}$ & $\begin{array}{l}\text { Patients with IBS; Medicare and } \\
\text { Medicaid HMO patients excluded [ACS] }\end{array}$ & $\begin{array}{l}\text { Controls matched for gender, } \\
\text { age, and number of comor- } \\
\text { bidities }\end{array}$ & United States & 501 & & & & \\
\hline \multicolumn{9}{|c|}{ Retrospective study (IBS only) } \\
\hline Brun-Strang $2007^{32}$ & $\begin{array}{l}\text { Patients meeting or not meeting Rome } \\
\text { II criteria }\end{array}$ & None & France & 452 & 121 & 120 & & \\
\hline ten Berg $2006^{35}$ & $\begin{array}{l}\text { Patients using mebeverine, a drug indi- } \\
\text { cated for IBS [ACS] }\end{array}$ & $\begin{array}{l}\text { Sample of the Dutch popula- } \\
\text { tion, U.S. population of IBS } \\
\text { patients }\end{array}$ & Netherlands & 136 & & & & \\
\hline Leong $2003^{17}$ & $\begin{array}{l}\text { Patients with IBS and a predominance of } \\
\text { constipation [ACS] }\end{array}$ & $\begin{array}{l}\text { Matched controls based on } \\
\text { age, sex, employment, job } \\
\text { classification, health plan, } \\
\text { and ZIP code }\end{array}$ & United States & 1,509 & & & & \\
\hline $\begin{array}{l}\text { Müller-Lissner } \\
2002^{36}\end{array}$ & Convenience sample of IBS patients & None & Germany & 200 & & & & \\
\hline Levy $2001^{24}$ & $\begin{array}{l}\text { Patients }>18 \text { years with a diagnosis of } \\
\text { IBS or irritable colon [ACS] }\end{array}$ & $\begin{array}{l}\text { Matched controls based on } \\
\text { birth year and sex, who did } \\
\text { not have IBD, GERD, or IBS }\end{array}$ & United States & 3,153 & & & & \\
\hline Ricci $2000^{20}$ & $\begin{array}{l}\text { Patients presenting with a primary } \\
\text { diagnosis of irritable colon (ICD-9-CM } \\
564.1 \text { ) [ACS] }\end{array}$ & $\begin{array}{l}\text { Cohort of asthma patients } \\
\text { matched by age and gender }\end{array}$ & United States & 2,770 & & & & \\
\hline Bentkover $1999^{37}$ & Patients $>20$ years with IBS & None & Canada & 118 & & & & \\
\hline \multicolumn{9}{|c|}{ Cross-sectional survey (IBS only) } \\
\hline Graham $2010^{38}$ & $\begin{array}{l}\text { Women veterans with IBS seeking care } \\
\text { at a VA clinic }\end{array}$ & Female veterans without IBS & United States & 113 & & & & \\
\hline Thijssen $2010^{39}$ & $\begin{array}{l}\text { Patients aged } 18-65 \text { with IBS according } \\
\text { to Rome II criteria }\end{array}$ & None & Netherlands & 268 & & & & \\
\hline Drossman 200940 & $\begin{array}{l}\text { Patients }>18 \text { years, diagnosed with IBS } \\
\text { by a physician }\end{array}$ & None & United States & 1,966 & 191 & 576 & 1,199 & \\
\hline Jeong $2008^{41}$ & $\begin{array}{l}\text { Patients with chronic GI symptoms } \\
\text { according to Rome II }\end{array}$ & General Korean population & Korea & 31 & 12 & 13 & 6 & \\
\hline Lee $2008^{42}$ & $\begin{array}{l}\text { Patients aged }>18 \text { with lower functional } \\
\text { abdominal symptoms }\end{array}$ & None & $\begin{array}{l}\text { United } \\
\text { Kingdom }\end{array}$ & 420 & & & & \\
\hline Amouretti $2006^{43}$ & $\begin{array}{l}\text { Adults > } 18 \text { years with IBS based on } \\
\text { Manning criteria, Rome I, or Rome II; } \\
\text { France }\end{array}$ & $\begin{array}{l}\text { Controls representative of } \\
\text { French population }\end{array}$ & France & 253 & 65 & 72 & 116 & \\
\hline Pare $2006^{44}$ & $\begin{array}{l}\text { Patients with abdominal pain and at least } \\
1 \text { symptom of bloating, constipation, or } \\
\text { alternating constipation and diarrhea }\end{array}$ & None & Canada & 1,555 & 457 & 196 & 433 & \\
\hline Dean $2005^{22}$ & $\begin{array}{l}\text { Single employer-based sample, IBS } \\
\text { based on Rome II criteria }\end{array}$ & None & United States & 720 & & & & \\
\hline Coffin 200445 & $\begin{array}{l}\text { Adults }>18 \text { years with IBS, attending } \\
\text { metropolitan outpatient clinic }\end{array}$ & None & France & 858 & 319 & 320 & 218 & \\
\hline Le Pen 200446 & $\begin{array}{l}\text { Patients aged }>18 \text { with IBS according to } \\
\text { Manning or Rome I or II criteria }\end{array}$ & None & France & 253 & 65 & 72 & 116 & \\
\hline
\end{tabular}




\section{TABLE 1 Overview of Selected Studies (continued)}

\begin{tabular}{|c|c|c|c|c|c|c|c|c|}
\hline \multirow[b]{2}{*}{ Reference } & \multirow[b]{2}{*}{ Population } & \multirow[b]{2}{*}{ Comparison Population } & \multirow{2}{*}{$\begin{array}{l}\text { Country, } \\
\text { Perspective }\end{array}$} & \multicolumn{5}{|c|}{ Number of Patients } \\
\hline & & & & IBS Total & IBS-C & IBS-D & IBS-A & $\mathrm{CC}$ \\
\hline Si 200447 & $\begin{array}{l}\text { Consecutively enrolled IBS patients } \\
\text { meeting Rome II criteria }\end{array}$ & None & China & 662 & 108 & 258 & 174 & \\
\hline Longstreth $2003^{25}$ & $\begin{array}{l}\text { Patients }>18 \text { years who had undergone } \\
\text { flexible sigmoidoscopy in the year } 2000\end{array}$ & Patients without IBS & United States & 578 & & & & \\
\hline Pace $2003^{48}$ & $\begin{array}{l}\text { Patients }>18 \text { years with ulcerative coli- } \\
\text { tis, Crohn's disease, or IBS }\end{array}$ & $\begin{array}{l}\text { Controls matched on sex } \\
\text { and age }\end{array}$ & United States & 85 & & & & \\
\hline Sandler $2002^{49}$ & $\begin{array}{l}\text { Patients with selected digestive diseases } \\
\text { drawn from national databases }\end{array}$ & None & United States & $\begin{array}{c}15,396,000 \\
\text { (prevalence } \\
1998)\end{array}$ & & & & \\
\hline Creed $2001^{50}$ & $\begin{array}{l}\text { Adults } 18-65 \text { years, meeting Rome cri- } \\
\text { teria, symptom duration }>6 \text { months, } \\
\text { failure to respond to usual medical } \\
\text { treatment, VAS }>59\end{array}$ & None & $\begin{array}{l}\text { United } \\
\text { Kingdom }\end{array}$ & 257 & 59 & 74 & 124 & \\
\hline Lee $2001^{51}$ & Consecutively screened IBS patients & None & United States & 714 & 141 & 195 & 378 & \\
\hline Gralnek $2000^{52}$ & $\begin{array}{l}\text { Aged }>18, \text { ambulatory, seen at UCLA } \\
\text { clinic }\end{array}$ & $\begin{array}{l}\text { U.S. general population, } \\
\text { published data }\end{array}$ & United States & 877 & & & & \\
\hline \multicolumn{9}{|c|}{ Retrospective study (IBS or CC) } \\
\hline Ahn $2008^{16}$ & $\begin{array}{l}\text { Sample of Medi-Cal population with } \\
\text { IBS+C using combination of ICD-9-CM } \\
\text { codes for constipation and IBS and } \\
\text { claims for constipation-related prescrip- } \\
\text { tions from } 1995 \text { to } 2003 \text { [ACS] }\end{array}$ & None & United States & & NR & & & NR \\
\hline Nyrop 200721 & $\begin{array}{l}\text { Patients aged } 18-75 \text { years, enrolled in } \\
\text { HMO for at least } 1 \text { year, no diagnosis } \\
\text { of ulcerative colitis or Crohn's disease } \\
\text { [ACS] }\end{array}$ & None & $\begin{array}{l}\text { United } \\
\text { States, HMO }\end{array}$ & 558 & & & & 203 \\
\hline \multicolumn{9}{|c|}{ Prospective study (CC only) } \\
\hline Pekmezaris $2002^{31}$ & $\begin{array}{l}\text { CC patients in a large metropolitan } \\
\text { skilled nursing facility }\end{array}$ & None & United States & & & & & 31 \\
\hline \multicolumn{9}{|c|}{ Case-control study (CC only) } \\
\hline Nguyen $2008^{27}$ & $\begin{array}{l}\text { Patients with a clinical diagnosis of con- } \\
\text { stipation (ICD-9-CM 564.0X) in the last } \\
\text { year [ACS] }\end{array}$ & $\begin{array}{l}\text { Patients without a clinical } \\
\text { diagnosis of constipation for } \\
\text { the previous } 5 \text { years }\end{array}$ & $\begin{array}{l}\text { United } \\
\text { States, HMO }\end{array}$ & & & & & 936 \\
\hline \multicolumn{9}{|c|}{ Cross-sectional survey (CC only) } \\
\hline Damon 200418 & $\begin{array}{l}\text { Patients with chronic constipation or } \\
\text { fecal incontinence referred to a hospital } \\
\text { for anorectal manometry }\end{array}$ & None & France & & & & & 78 \\
\hline \multicolumn{9}{|c|}{ 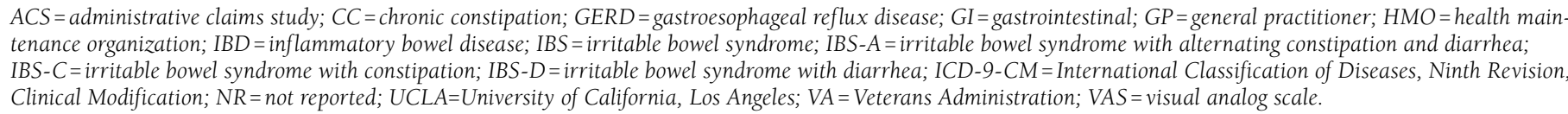 } \\
\hline
\end{tabular}

Nine studies were retrospective analyses of administrative claims databases, and in the remaining 7 studies, none analyzed cost outcomes by IBS subtypes. In 1 study using administrative claims data, 17 patients with IBS with constipation were identified using an algorithm that combined primary and secondary diagnosis codes (using International Classification of Diseases, Ninth Revision, Clinical Modification [CD-9-CM]) for irritable colon, abdominal pain, and constipation. This study estimated the annual direct cost of IBS to be $\$ 3,736$ (1998 USD). In a second study, 16 patients with constipation (C) or IBS co-occurring with constipation (termed IBS $+\mathrm{C}$ ) were identified from the California Medicaid population using a combination of ICD-9-CM codes for constipation and IBS and claims for constipation-related prescriptions. Per-patient total costs were \$1,912 for C and \$1,562 for IBS + C (2002 USD). All costs for all categories studied were similar between the $C$ and IBS $+C$ groups over the 6-year study period $(P>0.05)$.

From the U.S. perspective, the indirect cost per patient of IBS ranged from $\$ 791$ per year for IBS-C (1998 USD; \$1,356 per year in 2012 USD) to $\$ 7,737$ for general IBS (year NR, study published in 2005; $\$ 9,933$ per year in 2012 USD). Indirect cost was estimated as lost productivity due to IBS symptoms or health care-seeking behaviors. No studies assessed the indirect costs of CC (Table 2). 


\section{TABLE 2 Economic Burden of Disease in Irritable Bowel Syndrome or Chronic Constipation}

\begin{tabular}{|c|c|c|c|c|c|c|c|}
\hline \multirow[b]{2}{*}{ Reference } & \multirow[b]{2}{*}{ Condition } & \multirow{2}{*}{$\begin{array}{c}\text { Country, } \\
\text { Perspective }\end{array}$} & \multicolumn{2}{|c|}{ Distribution of Costs (\% of Total) ${ }^{a}$} & \multicolumn{2}{|c|}{ Total Costs } & \multirow[b]{2}{*}{ Units } \\
\hline & & & Direct & Indirect & Direct & Indirect & \\
\hline $\begin{array}{l}\text { Johansson } \\
2010^{30}\end{array}$ & IBS & Norway & $\begin{array}{l}\text { Consultations (16.1\%), hospitalizations ( } 67.7 \%) \text {, } \\
\text { medications (4.5\%), and alternative products } \\
(2.5 \%)\end{array}$ & & $1,049 \mathrm{NOK}$ & & $\begin{array}{l}2001 ; \\
€ 1=\text { NOK } 8\end{array}$ \\
\hline $\begin{array}{l}\text { Brun- } \\
\text { Strang } \\
2007^{32}\end{array}$ & IBS & France & $\begin{array}{l}\text { Physician consultations, medical fees, hospital- } \\
\text { izations, ambulatory examinations, examina- } \\
\text { tions, medications, drug costs (\% NR) }\end{array}$ & $\begin{array}{l}\text { Mean number of sick } \\
\text { leave days, restriction } \\
\text { of activities (\% NR) }\end{array}$ & $€ 756.14$ & $€ 37.89$ & $2001 €$ \\
\hline $\begin{array}{l}\text { Dean } \\
2005^{22}\end{array}$ & IBS & $\begin{array}{l}\text { United } \\
\text { States }\end{array}$ & & $\begin{array}{l}\text { Work productivity loss } \\
\text { due to IBS-attributable } \\
\text { symptoms (100\%) }\end{array}$ & & $\begin{array}{l}\$ 7,737 \\
{[\$ 9,933]}\end{array}$ & $\begin{array}{l}\text { NR, published } \\
\text { in } 2005 \\
\text { [2012 USD] } \\
\end{array}$ \\
\hline $\begin{array}{l}\text { Le Pen } \\
200446\end{array}$ & IBS & France & $\begin{array}{l}\text { Consultations ( } 18.6 \%) \text {, tests and investigations } \\
(40.6 \%) \text {, hospitalizations ( } 26.4 \%) \text {, and medica- } \\
\text { tions (14.4\%) }\end{array}$ & $\begin{array}{l}\text { Cost of absences from } \\
\text { work }(100 \%)\end{array}$ & $€ 47.3 /$ month & $€ 3.3 /$ month & $1999-2000 €$ \\
\hline $\begin{array}{l}\text { Leong } \\
2003^{17}\end{array}$ & IBS & $\begin{array}{l}\text { United } \\
\text { States }\end{array}$ & $\begin{array}{l}\text { Hospital outpatient ( } 33.7 \%) \text {, hospital inpatient } \\
(21.7 \%) \text {, physician's office }(14 \%) \text {, other medical } \\
(2.2 \%) \text {, and pharmacy }(28.4 \%)\end{array}$ & $\begin{array}{l}\text { Disability (61.9\%) and } \\
\text { absenteeism (38.1\%) }\end{array}$ & $\begin{array}{l}\$ 3,736^{b} \\
{[\$ 6,403]}\end{array}$ & $\begin{array}{c}\$ 791 \\
{[\$ 1,356]}\end{array}$ & $\begin{array}{c}1998 \text { USD } \\
{[2012 \text { USD] }}\end{array}$ \\
\hline $\begin{array}{l}\text { Longstreth } \\
2003^{25}\end{array}$ & IBS & $\begin{array}{l}\text { United } \\
\text { States }\end{array}$ & $\begin{array}{l}\text { Outpatient }(52.6 \%) \text {, inpatient (9.8\%), pharmacy } \\
(28.3 \%) \text {, radiology }(5.1 \%) \text {, and laboratory }(4.2 \%)\end{array}$ & & $\begin{array}{l}\$ 3,729.04 \mathrm{~b} \\
{[\$ 5,932.78]}\end{array}$ & & $\begin{array}{c}2000 \text { USD } \\
{[2012 \text { USD] }}\end{array}$ \\
\hline $\begin{array}{l}\text { Martin } \\
2003^{26}\end{array}$ & IBS & $\begin{array}{l}\text { United } \\
\text { States } \\
\text { (CA or } \\
\text { NC), } \\
\text { Medicaid }\end{array}$ & $\begin{array}{l}\text { (CA, NC) Prescriptions }(46.6 \%, 23.3 \%) \text {, phy- } \\
\text { sician visit }(19.2 \%, 13.9 \%) \text {, inpatient }(6.2 \% \text {, } \\
23.9 \%) \text {, outpatient }(7 \%, 20.3 \%) \text {, and other costs } \\
(21 \%, 18.6 \%)\end{array}$ & & $\begin{array}{l}\text { PMPY CA: } \\
\$ 2,952^{\mathrm{b}} \\
{[\$ 4,123]} \\
\text { PMPY NC: } \\
\$ 5,906^{\mathrm{b}} \\
{[\$ 8,248]}\end{array}$ & & $\begin{array}{l}\text { NR, published } \\
\quad \text { in } 2003 \\
\text { [2012 USD] }\end{array}$ \\
\hline $\begin{array}{l}\text { Akehurst } \\
2002^{23}\end{array}$ & IBS & $\begin{array}{l}\text { United } \\
\text { Kingdom }\end{array}$ & $\begin{array}{l}\text { Outpatient (25.3\%), inpatient (38.1\%), accident } \\
\text { and emergency visits ( } 2.1 \%) \text {, prescription medi- } \\
\text { cations }(20.3 \%) \text {, home visits }(0.5 \%) \text {, GP surgery } \\
\text { attendances }(13.8 \%)\end{array}$ & & $£ 316.2^{\mathrm{b}}$ & & $1997 / 1998 £$ \\
\hline $\begin{array}{l}\text { Müller- } \\
\text { Lissner } \\
2002^{36}\end{array}$ & IBS & Germany & $\begin{array}{l}\text { Physician costs }(25 \%) \text {, drug costs }(46 \%) \text {, psycho- } \\
\text { therapy }(2 \%) \text {, other procedures }(<1 \%) \text {, hospital } \\
(25 \%) \text {, and rehabilitation }(2 \%)\end{array}$ & Sick leave (100\%) & $€ 791.48$ & $€ 398$ & $1998 €$ \\
\hline $\begin{array}{l}\text { Patel } \\
2002^{28}\end{array}$ & IBS & $\begin{array}{l}\text { United } \\
\text { States }\end{array}$ & $\begin{array}{l}\text { Laboratory, procedures, drug, emergency depart- } \\
\text { ment, outpatient, and inpatient costs (\% NR) }\end{array}$ & & $\begin{array}{l}\$ 2,504 \mathrm{~b} \\
{[\$ 4,429]}\end{array}$ & & $\begin{array}{c}\text { 1997-1999 USD } \\
\text { [2012 USD] }\end{array}$ \\
\hline $\begin{array}{l}\text { Sandler } \\
2002^{49}\end{array}$ & IBS & $\begin{array}{l}\text { United } \\
\text { States }\end{array}$ & $\begin{array}{l}\text { Hospital facility (62.7\%), inpatient physician } \\
\text { services (11\%), hospital outpatient department } \\
(2.6 \%) \text {, hospital emergency room (1\%), office vis- } \\
\text { its }(16.9 \%) \text {, and drugs }(5.9 \%)\end{array}$ & $\begin{array}{l}\text { Work loss secondary } \\
\text { to receiving health } \\
\text { care }(100 \%)\end{array}$ & $\begin{array}{c}\text { Total U.S.: } \\
\$ 1,353 \mathrm{M} \\
{[\$ 2,319 \mathrm{M}]}\end{array}$ & $\begin{array}{l}\text { Total U.S.: } \\
\$ 205 \mathrm{M} \\
{[\$ 351 \mathrm{M}]}\end{array}$ & $\begin{array}{c}1998 \text { USD } \\
{[2012 \text { USD] }}\end{array}$ \\
\hline $\begin{array}{l}\text { Creed } \\
2001^{50}\end{array}$ & IBS & England & $\begin{array}{l}\text { Hospital care }(76.8 \%) \text {, primary care costs } \\
(23.5 \%) \text {, alternative therapies }(0.2 \%) \text {, patient } \\
\text { costs }(3.6 \%) \text {, and community costs }(0.9 \%)\end{array}$ & $\begin{array}{l}\text { Lost time from work } \\
(100 \%)\end{array}$ & $\begin{array}{l}\$ 1,743 \\
{[\$ 2,651]}\end{array}$ & $\begin{array}{l}\$ 334.50 \\
{[\$ 508.77]} \\
\end{array}$ & $\begin{array}{l}\text { NR, published } \\
\text { in } 2003 \\
\text { [2012 USD] } \\
\end{array}$ \\
\hline $\begin{array}{l}\text { Levy } \\
2001^{24}\end{array}$ & IBS & $\begin{array}{l}\text { United } \\
\text { States }\end{array}$ & $\begin{array}{l}\text { Inpatient care }(24.7 \%) \text {, primary care visits } \\
(19.6 \%) \text {, specialty care }(15.9 \%) \text {, prescription } \\
\text { medicines }(12.3 \%) \text {, radiology }(7.8 \%) \text {, mental } \\
\text { health care }(2.9 \%) \text {, laboratory tests }(2.9 \%) \text {, emer- } \\
\text { gency visits }(2.8 \%) \text {, and other costs }(9.6 \%)\end{array}$ & & $\begin{array}{l}\$ 4,044 b \\
{[\$ 7,610]}\end{array}$ & & $\begin{array}{l}1995 \text { USD } \\
{[2012 \text { USD] }}\end{array}$ \\
\hline $\begin{array}{l}\text { Ricci } \\
2000^{20}\end{array}$ & IBS & $\begin{array}{l}\text { United } \\
\text { States }\end{array}$ & $\begin{array}{l}\text { Outpatient charges }(27.3 \%) \text {, pharmacy charges } \\
(8.0 \%) \text {, inpatient charges }(40.8 \%) \text {, ancillary } \\
\text { charges }(23.9 \%)\end{array}$ & & $\begin{array}{r}\$ 7,547 \\
{[\$ 12,007]} \\
\end{array}$ & & $\begin{array}{l}\text { NR, published } \\
\text { in } 2000 \\
\text { [2012 USD] } \\
\end{array}$ \\
\hline $\begin{array}{l}\text { Bentkover } \\
199937\end{array}$ & IBS & Canada & $\begin{array}{l}\text { Doctors' fees ( } 18 \%-29 \%) \text {, diagnostic tests ( } 45 \% \text { - } \\
46 \% \text { ), drugs ( } 5 \%-19 \%) \text {, medical professional } \\
\text { referrals }(10 \%) \text {, emergency room visits ( } 7 \%-11 \%)\end{array}$ & $\begin{array}{l}\text { Lost time at work due } \\
\text { to IBS }(100 \%)\end{array}$ & $\$ 258.82$ & $\$ 748.16$ & 1996 CAD \\
\hline $\begin{array}{l}\text { Ahn } \\
2008^{16}\end{array}$ & $\mathrm{IBS}+\mathrm{C}, \mathrm{CC}$ & $\begin{array}{l}\text { United } \\
\text { States }\end{array}$ & $\begin{array}{l}\text { (C/IBS+C:) outpatient }(53.8 \%, 62.3 \%) \text {, inpatient } \\
(18.3 \%, 13.4 \%) \text {, drugs }(22.3 \%, 22.6 \%) \text {, and long- } \\
\text { term care }(5.8 \%, 1.7 \%)\end{array}$ & & $\begin{array}{c}\text { C } \$ 1,912 \\
{[\$ 2,778]} \\
\text { IBS+C } \\
\$ 1,562 \\
{[\$ 2,269]}\end{array}$ & & $\begin{array}{c}2002 \text { USD } \\
\text { [2012 USD] }\end{array}$ \\
\hline $\begin{array}{l}\text { Nyrop } \\
2007^{21}\end{array}$ & IBS, CC & $\begin{array}{l}\text { United } \\
\text { States, } \\
\text { HMO }\end{array}$ & $\begin{array}{l}\text { (IBS/C) inpatient (14.2\%, 22.9\%), primary care } \\
\text { office visit (19.8\%, } 17.1 \%) \text {, GI clinic office visit } \\
(12.8 \%, 12.6 \%) \text {, mental health office visit ( } 3.5 \% \text {, } \\
1.5 \%) \text {, pharmacy }(17.7 \%, 13.9 \%) \text {, radiology } \\
(7.6 \%, 5.8 \%) \text {, emergency }(2.6 \%, 3.5 \%) \text {, lab }\end{array}$ & & $\begin{array}{l}\text { C } \$ 7,522 \\
{[\$ 11,967]} \\
\text { IBS } \$ 5,049 \\
{[\$ 8,033]}\end{array}$ & & $\begin{array}{c}\text { 2000-2003 USD } \\
\text { [2012 USD] }\end{array}$ \\
\hline
\end{tabular}


TABLE 2 Economic Burden of Disease in Irritable Bowel Syndrome or Chronic Constipation (continued)

\begin{tabular}{|c|c|c|c|c|c|c|c|}
\hline \multirow[b]{2}{*}{ Reference } & \multirow[b]{2}{*}{ Condition } & \multirow{2}{*}{$\begin{array}{c}\text { Country, } \\
\text { Perspective }\end{array}$} & \multicolumn{2}{|c|}{ Distribution of Costs (\% of Total) ${ }^{a}$} & \multicolumn{2}{|c|}{ Total Costs } & \multirow[b]{2}{*}{ Units } \\
\hline & & & Direct & Indirect & Direct & Indirect & \\
\hline $\begin{array}{l}\text { Nguyen } \\
2008^{27}\end{array}$ & $\mathrm{CC}$ & $\begin{array}{l}\text { United } \\
\text { States, } \\
\text { HMO }\end{array}$ & $\begin{array}{l}\text { Outpatient visits, GI outpatient visits, non-GI } \\
\text { outpatient visits (\% NR) }\end{array}$ & & $\$ 7,510^{\mathrm{b}}$ & & NR \\
\hline $\begin{array}{l}\text { Pekmezaris } \\
2002^{31}\end{array}$ & $\mathrm{CC}$ & $\begin{array}{l}\text { United } \\
\text { States }\end{array}$ & $\begin{array}{l}\text { Constipation medication, medications not related } \\
\text { to care of constipation, constipation work-up, } \\
\text { time required to deliver constipation care, labor } \\
\text { rates for constipation care, medication costs (\% } \\
\text { NR; staff time accounted for more than the cost } \\
\text { of all drugs administered) }\end{array}$ & & $\begin{array}{c}\$ 61.18 / \text { day } \\
{[\$ 101.30 / \text { day }]}\end{array}$ & & $\begin{array}{l}1999 \text { USD } \\
\text { [2012 USD] }\end{array}$ \\
\hline
\end{tabular}

a All costs are annual costs, unless otherwise specified. Values in brackets are costs inflated to 2012 USD using the U.S. city average, medical care Consumer Price Index, and 1984 as the base year (http://www.bls.gov/data). Costs for countries other than the United States were not converted to USD or inflated.

${ }^{b}$ Costs significantly higher in IBS or CC patients compared with matched non-IBS or non-CC controls.

$C A=$ California; $C A D=$ Canadian dollar; $C C=$ chronic constipation; $G I=$ gastrointestinal; $G P=$ general practitioner; $H M O=$ health maintenance organization; IBS = irritable bowel syndrome; $M=$ million; $N C=$ North Carolina; $N O K=$ Norwegian kroner; $N R=$ not reported; $P M P Y=$ per member per year; USD=U.S. dollar.

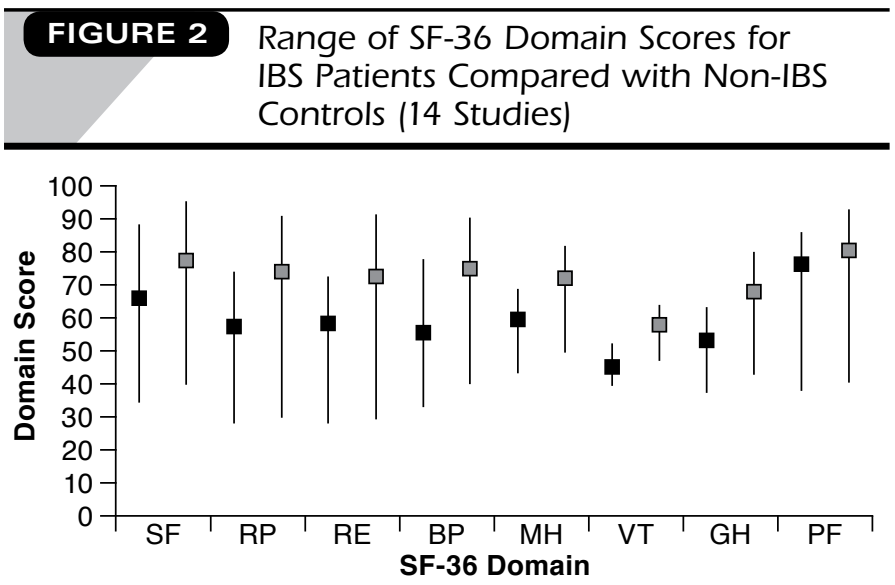

Mean, patients with IBS $\square$ Mean, controls without IBS

$\mathrm{BP}=$ bodily pain; $\mathrm{GH}=$ general health; $\mathrm{IBS}=$ irritable bowel syndrome; $\mathrm{MH}=$ mental health; $P F=$ physical functioning; $R E=$ role emotional; $R P=$ role physical; $S F=$ social functioning; $V T=$ vitality.

\section{Studies Assessing Humanistic Burden of Disease}

Nineteen studies meeting the inclusion criteria measured humanistic burden of disease using the following instruments: Short Form (36) Health Survey (SF-36; n=14), EuroQol 5D health questionnaire (EQ-5D; $n=1)$, Irritable Bowel Syndrome Quality of Life (IBS-QOL; $n=6$ ) or Gastrointestinal Quality of Life Index (GIQLI; $n=1$ ). Several studies used multiple instruments. Six studies investigated patients in the United States; 4 studied patients in France; 2 each studied patients in the United Kingdom, Korea, and the Netherlands; and 1 each studied patients in Sweden, Canada, and China.

Instruments used to study QOL may be generic or disease specific, and SF-36 is a widely used generic measure of health status. All of the studies that measured SF-36 domain scores were in patients with IBS. In the studies that compared IBS with a non-IBS control population, SF-36 domain scores were significantly lower in IBS patients than in controls (Figure 2). The difference in minimum and maximum domain scores was smallest in the domain of vitality (16.7 points) and largest in the domain of role-emotional (62.2 points). None of these studies reported SF-36 scores by IBS subtype.

IBS-QOL is a condition-specific measure for assessing HRQOL among persons with IBS. A total of 6 studies in IBS patients measured QOL using the IBS-QOL. Variation in IBSQOL domain scores across studies was less than SF-36 domain scores: the smallest score range was in the health worry domain (10.5 points), while the largest range was seen in the food avoidance (35.9 points) domain (Figure3).

Only 1 study of CC patients that reported humanistic burden of disease met the inclusion criteria. ${ }^{18}$ This study included a total of 78 patients with CC and measured QOL using the GIQLI, an instrument developed for gastrointestinal and digestive disorders. The mean total GIQLI score for CC patients was $92.3 \pm 24.8$, which was lower than the mean total score for healthy subjects $(126 \pm 18) .{ }^{19}$

\section{Discussion}

This systematic review identified studies assessing the economic and humanistic burden of IBS and/or CC. A total of 35 studies met the inclusion criteria and were analyzed. The main findings were as follows: (a) estimates of the economic burden of disease vary widely by about a factor of 5 for direct costs and nearly a factor of 10 for indirect costs; (b) there is an overall lack of uniformity in methods used for patient identification and measurement of costs; (c) few studies have attempted to distinguish among IBS subtypes or between CC and IBS-C; and (d) few recent studies have been published. 


\section{FIGURE 3 Range of IBS-OOL Total and Domain} Scores for IBS Patients (6 Studies)

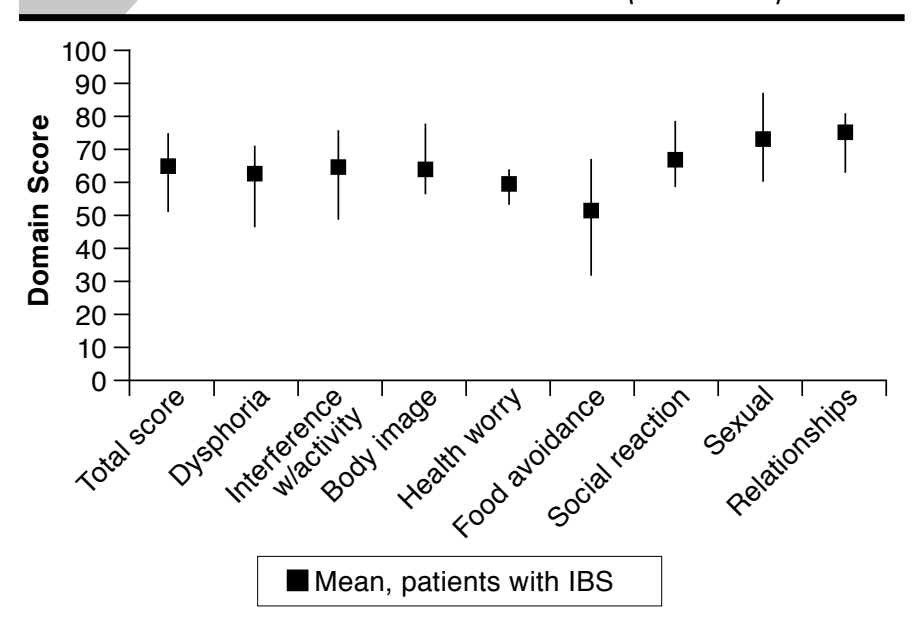

IBS = irritable bowel syndrome.

This systematic review found a wide range of cost estimates for both IBS and CC. From the U.S. perspective, the direct costs of IBS and CC ranged nearly 5-fold from the lower estimates $\left(\$ 1,562\right.$ per year ${ }^{16}$ for IBS and $\$ 1,912$ per year ${ }^{16}$ for CC; both 2002 USD) to the upper estimates $\left(\$ 7,547\right.$ per year $^{20}$ [year NR] for IBS and \$7,522 per year ${ }^{21}$ for CC [2002-2003 USD]). Indirect per-patient costs of IBS from the U.S. perspective ranged from $\$ 791$ per year ${ }^{17}$ to $\$ 7,737$ per year. ${ }^{22}$ Notably, in studies comparing IBS or CC patients with controls without these FGIDs, costs were significantly higher in IBS or CC patients (Table 2) ${ }^{17,23-28}$ There are a number of possible reasons for this wide variation. Methodological differences such as definitions of IBS or CC, methods for identifying patients with these conditions, valuation years, regional cost differences, and different categories of service studied are likely factors.

In studies assessing humanistic burden of disease, identification of patients was still highly variable, but standard approaches were used to measure the QOL burden of disease. A total of 14 studies collected SF-36 scores in patients with IBS, 8 of which included non-IBS controls. In these 8 studies, SF-36 domain scores were consistently and significantly poorer in patients with IBS compared with matched non-IBS controls (Figure 2). Overall, SF-36 domain scores in patients with IBS varied widely, but scores for the domain of vitality appeared to be particular low and tightly grouped, with a mean score of 57.9 points and intra-domain range of 16.7 points (Figure 2). In the 6 studies that measured IBS-QOL scores, domain scores were more tightly grouped, but the average range between minimum and maximum domain scores was still approximately 25 points (Figure 3). No study reported QOL scores within specific IBS subtypes. Only 1 study included evaluated QOL in patients with CC and found diminished QOL as measured by the GIQLI compared with healthy controls.

The difficulty in differentially identifying patients with IBS subtypes or CC has meaningful consequences for clinical practices and optimal treatment selection in a managed care setting. Decrements in functioning and pain for patients with IBS subtypes may differ, which could inform treatment selection. Findings from this systematic review suggest that patients with IBS suffer from decreased vitality, but differences in symptom burden and the impact on patients among IBS subtypes have apparently not yet been published outside the context of particular interventions. Results also reveal significant QOL burdens in IBS patients and highlight the need for future studies to investigate the QOL burdens specific to IBS subtypes and CC. A better understanding of patients' clinical experience within FGID subtypes is essential for selecting appropriate therapy.

A lack of diagnostic consensus for IBS or CC was also observed, which prevented rigorous comparisons of results across studies. In the claims database studies, no consistent set of ICD-9-CM codes was used to identify patients with IBS or CC, and the 2 studies that focused on patients with cooccurrence of constipation and IBS used markedly different technical approaches. In general, studies did not differentiate among IBS subtypes.

This analysis revealed considerable gaps in the literature measuring the burden of disease for IBS and CC. The aim of this systematic literature review was to estimate the burden of disease in 2 common gastrointestinal disorders, with particular focus on IBS subtypes, but few studies that attempted to parse findings by specific FGID were found. Only 2 studies examined the costs of patients with IBS-C. ${ }^{16,17}$ There were no studies that examined the humanistic burden associated with any IBS subtype, and only 3 of the included studies investigated cost or QOL burdens in patients with CC. There were no studies estimating the economic burden of these gastrointestinal disorders in recent $\left(<5\right.$ years) values, and the only study ${ }^{18}$ that estimated the humanistic burden of CC used a comparison cohort of patients assessed in 1999. Finally, current treatment patterns for these FGIDs have not been documented extensively.

Previous systematic reviews assessing burden of disease have focused on the effect of IBS on HRQOL ${ }^{13,29}$ and direct resource utilization and productivity losses, ${ }^{14}$ whereas no systematic reviews documenting the burden of disease for CC have been recently published. This systematic review aimed to identify burden-of-illness studies for IBS and CC and focus on their subtypes. Findings suggest that there is a lack of information regarding the economic and humanistic burden of CC and IBS subtypes. This variation can be attributed to the difficulty in identifying patients with IBS subtypes and CC in retrospective studies and, particularly for economic studies, to the lack of consistency in methods used to measure direct or indirect health care costs. 


\section{Limitations}

This systematic review has several important limitations. First, differences in disease definitions according to studies' ICD9-CM criteria and lack of similarities in patient populations precluded meta-analysis of the results, which would have allowed us to estimate disease costs and humanistic burden. Second, the design of studies included in the systematic review varied considerably. Of the 35 included studies, only $2^{30,31}$ were prospectively conducted, while the remainder were case control (6), retrospective (9), or cross-sectional (18) in design. It was appropriate to include these study designs in the systematic review, since the stated criteria excluded studies that reported economic or humanistic outcomes attributable to a particular intervention (which eliminated many interventional studies). Third, the cost estimates used in the economic analyses were often dated: the most recent valuation period for direct costs was for 2000-2003, ${ }^{21}$ while for indirect costs the most recent values were for 2001. ${ }^{32}$ This hindered the ability to provide a precise estimate of the current economic burden of disease for IBS, IBS subtypes, and CC. Furthermore, studies used a range of international currencies and evaluated costs using different index years, which made it difficult to compare the cost estimates observed in the systematic review. This review included studies of varying rigor, including both abstracts and full publications, and the studies were not evaluated for quality. These limitations do not alter the main findings already described.

\section{Conclusions}

This systematic review included a total of 35 studies evaluating the economic or humanistic burden of disease of IBS or CC. In the 19 studies that assessed the costs of these FGIDs, 15 included patients with IBS only, 2 included patients with CC only, and 2 included patients with either IBS or CC. From the U.S. perspective, the direct costs of IBS ranged from $\$ 1,562$ to $\$ 7,547$ per year, and the indirect costs ranged from $\$ 791$ to $\$ 7,737$ per year, while the direct costs of $C C$ ranged from $\$ 1,912$ to $\$ 7,522$ per year (indirect costs for CC were not observed in this systematic review). The humanistic burden of illness of IBS patients compared with matched controls was demonstrated in multiple studies using SF-36 and IBS-QOL scores; however, only 1 study that met our inclusion criteria assessed the burden of illness in CC patients, observing a diminished QOL compared with non-CC controls. Our review did not identify any studies that used standard diagnostic criteria to assess the economic or humanistic burden of IBS subtypes or CC. Given the limited information regarding economic and humanistic burden in these 2 common FGID conditions, future research should focus on characterizing the burden of illness associated with IBS subtypes and CC.

\section{Authors}

DAVE NELLESEN, PhD, MBA, is Vice President; KIMBERLY YEE, MPH, is Senior Analyst; and ANITA CHAWLA, PhD, is Managing Principal, Analysis Group, Inc., Menlo Park, California. BARBARA EDELMAN LEWIS, PhD, MHA, was Senior Director, Health Economics \& Outcomes Research, Ironwood Pharmaceuticals, Inc., Cambridge, Massachusetts, at the time this research was conducted and is currently a Health Economics \& Outcomes Research Consultant, Regeneron Pharmaceuticals, Inc., Tarrytown, New York. ROBYN T. CARSON, MPH, is Associate Director, Health Economics $\&$ Outcomes Research, Forest Research Institute, Forest Laboratories, Inc., Jersey City, New Jersey.

AUTHOR CORRESPONDENCE: Dave Nellesen, PhD, MBA,

1010 El Camino Real, Ste. 310, Menlo Park, CA 94025.

Tel.: 650.463.2703; Fax: 650.323.2796;

E-mail:dnellesen@analysisgroup.com.

\section{DISCLOSURES}

This study was conducted by Analysis Group, Inc., which received consultancy fees from Forest Laboratories, Inc., and Ironwood Pharmaceuticals, Inc. Forest Laboratories, Inc.; Ironwood Pharmaceuticals, Inc.; and Analysis Group, Inc., were involved in the study design, literature review and interpretation, and the decision to submit these data for publication. Funds for preparation of this manuscript were provided by Ironwood Pharmaceuticals, Inc., and Forest Laboratories, Inc.

At the time of the study, Lewis was an employee of Ironwood Pharmaceuticals, Inc. Carson is an employee of Forest Research Institute and owns stock/stock options in Forest Laboratories. Nelleson and Chawla are employees of Analysis Group, Inc., and Yee was an employee of Analysis Group at the time of the study.

Nellesen, Yee, Chawla, Carson, and Lewis conceived and designed the research; Yee performed the analysis; and Nellesen, Yee, Chawla, Lewis, and Carson prepared the manuscript.

\section{REFERENCES}

1. Drossman DA. The functional gastrointestinal disorders and the Rome III process. Gastroenterology. 2006;130(5):1377-90.

2. American Gastroenterological Association. American Gastroenterological Association medical position statement: irritable bowel syndrome. Gastroenterology. 2002;123(6):2105-07

3. Locke GR 3rd, Pemberton JH, Phillips SF. American Gastroenterological Association medical position statement: guidelines on constipation. Gastroenterology. 2000;119(6):1761-66.

4. Longstreth GF, Thompson WG, Chey WD, Houghton LA, Mearin F, Spiller RC. Functional bowel disorders. Gastroenterology. 2006;130(5):1480-91.

5. Hungin A, Chang L, Locke G, Dennis EH, Barghout V. Irritable bowel syndrome in the United States: prevalence, symptom patterns and impact. Aliment Pharmacol Ther. 2005;21(11):1365-75.

6. Mayer E, Naliboff B, Lee O, Munakata J, Chang L. Review article: gender related differences in functional gastrointestinal disorders. Aliment Pharmacol Ther. 1999;13(Suppl 2):S65-S69.

7. Whitehead W, Palsson O, Jones K. Systematic review of the comorbidity of irritable bowel syndrome with other disorders: what are the causes and implications? Gastroenterology. 2002;122(4):1140-56.

8. Harris L, Hansel S, DiBaise J, Crowell MD. Irritable bowel syndrome and chronic constipation: emerging drugs, devices, and surgical treatments. Curr Gastroenterol Rep. 2006;8(4):282-90.

9. Brandt LJ, Prather CM, Quigley EM, Schiller LR, Schoenfeld P, Talley NJ. Systematic review on the management of chronic constipation in North America. Am J Gastroenterol. 2005;100(Suppl 1):S5-S21 
10. Johanson JF, Kralstein J. Chronic constipation: a survey of the patient perspective. Aliment Pharmacol Ther. 2007;25(5):599-608.

11. Choung R, Locke GR 3rd, Schleck CD, Zinsmeister AR, Talley NJ. Cumulative incidence of chronic constipation: a population-based study 1988-2003. Aliment Pharmacol Ther. 2007;26(11-12):1521-28.

12. Guilera M, Balboa A, Mearin F. Bowel habit subtypes and temporal patterns in irritable bowel syndrome: systematic review. Am J Gastroenterol. 2005;100(5):1174-84.

13. El-Serag HB, Olden K, Bjorkman D. Health-related quality of life among persons with irritable bowel syndrome: a systematic review. Aliment Pharmacol Ther. 2002;16(6):1171-85.

14. Inadomi JM, Fennerty MB, Bjorkman D. Systematic review: the economic impact of irritable bowel syndrome. Aliment Pharmacol Ther. 2003;18(7):671-82. 15. Hulisz D. The burden of illness of irritable bowel syndrome: current challenges and hope for the future. J Manag Care Pharm. 2004;10(4):299-309. Available at: http://www.amcp.org/WorkArea/DownloadAsset.aspx?id=7003 16. Ahn J, Brook R, Nichol B, et al. Similarities between constipation with and without irritable bowel syndrome in a California Medicaid (Medi-Cal) population: costs trends by category in the 12 months after diagnosis from 1997-2002. Gastroenterology. 2008;134(4):A-468.

17. Leong SA, Barghout V, Birnbaum HG, et al. The economic consequences of irritable bowel syndrome: a U.S. employer perspective. Arch Intern Med. 2003;163(8):929-35.

18. Damon H, Dumas P, Mion F. Impact of anal incontinence and chronic constipation on quality of life. Gastroenterol Clin Biol. 2004;28(1):16-20.

19. Slim K, Bousquet J, Kwiatkowski F, et al. Premiere validation de la version fracaise de l'index de qualite de vie pour les maladies digestives (GIQLI) [First validation of the French version of the Gastrointestinal Quality of Life Index (GIQLI)]. Gastroenterol Clin Biol. 1999;23(1):25-31. 20. Ricci J, Jhingran P, McLaughlin T, Carter EG. Costs of care for irritable bowel syndrome in managed care. J Clin Outcomes Manag. 2000;7(6):23-28 21. Nyrop KA, Palsson OS, Levy RL, et al. Costs of health care for irritable bowel syndrome, chronic constipation, functional diarrhoea and functional abdominal pain. Aliment Pharmacol Ther. 2007;26(2):237-48.

22. Dean BB, Aguilar D, Barghout V, et al. Impairment in work productivity and health-related quality of life in patients with IBS. Am J Manag Care. 2005;11(1 Suppl):S17-S26.

23. Akehurst RL, Brazier JE, Mathers N, et al. Health-related quality of life and cost impact of irritable bowel syndrome in a UK primary care setting. Pharmacoeconomics. 2002;20(7):455-62.

24. Levy RL, Von Korff M, Whitehead WE, et al. Costs of care for irritable bowel syndrome in patients in a health maintenance organization. Am J Gastroenterol. 2001;96(11):3122-29.

25. Longstreth GF, Wilson A, Knight K, et al. Irritable bowel syndrome, health care use, and costs: a U.S. managed care perspective. Am J Gastroenterol. 2003;98(3):600-07.

26. Martin BC, Ganguly R, Pannicker S, Frech F, Barghout V. Utilization patterns and net direct medical cost to Medicaid of irritable bowel syndrome. Curr Med Res Opin. 2003;19(8):771-80.

27. Nguyen T, Palsson O, Von Korff M, et al. Health care costs and utilization in patients with chronic constipation. Gastroenterology. 2008;134 (4 Suppl 1):A280.

28. Patel RP, Petitta A, Fogel R, Peterson E, Zarowitz BJ. The economic impact of irritable bowel syndrome in a managed care setting. J Clin Gastroenterol. 2002;35(1):14-20.

29. El-Serag HB. Impact of irritable bowel syndrome: prevalence and effect on health-related quality of life. Rev Gastroenterol Disord. 2003;3(Suppl 2):S3-S11. 30. Johansson PA, Farup PG, Bracco A, Vandvik PO. How does comorbidity affect cost of health care in patients with irritable bowel syndrome? A cohort study in general practice. BMC Gastroenterol. 2010;10:31.

31. Pekmezaris R, Aversa L, Wolf-Klein G, Cedarbaum J, Reid-Durant M. The cost of chronic constipation. J Am Med Dir Assoc. 2002;3(4):224-28.

32. Brun-Strang C, Dapoigny M, Lafuma A, Wainsten JP, Fagnani F. Irritable bowel syndrome in France: quality of life, medical management, and costs: the Encoli study. Eur J Gastroenterol Hepatol. 2007;19(12):1097-103.
33. Park JM, Choi MG, Kim YS, et al. Quality of life of patients with irritable bowel syndrome in Korea. Qual Life Res. 2009;18(4):435-46.

34. Faresjö A, Grodzinsky E, Johansson S, Wallander MA, Timpka T, Akerlind I. A population-based case-control study of work and psychosocial problems in patients with irritable bowel syndrome-women are more seriously affected than men. Am J Gastroenterol. 2007;102(2):371-79.

35. ten Berg MJ, Goettsch WG, van den Boom G, Smout AJ, Herings RM. Quality of life of patients with irritable bowel syndrome is low compared to others with chronic diseases. Eur J Gastroenterol Hepatol. 2006;18(5):475-81. 36. Müller-Lissner SA, Pirk O. Irritable bowel syndrome in Germany. A cost of illness study. Eur J Gastroenterol Hepatol. 2002;14(12):1325-29.

37. Bentkover JD, Field C, Greene EM, Plourde V, Casciano JP. The economic burden of irritable bowel syndrome in Canada. Can J Gastroenterol. 1999;13(Suppl A):S89-S96.

38. Graham DP, Savas L, White D, et al. Irritable bowel syndrome symptoms and health-related quality of life in female veterans. Aliment Pharmacol Ther. 2010;31(2):261-73.

39. Thijssen AY, Jonkers DM, Leue C, et al. Dysfunctional cognitions, anxiety and depression in irritable bowel syndrome. J Clin Gastroenterol. 2010;44(10):e236-e241

40. Drossman DA, Morris CB, Schneck S, et al. International survey of patients with IBS: symptom features and their severity, health status, treatments, and risk taking to achieve clinical benefit. J Clin Gastroenterol. 2009;43(6):541-50.

41. Jeong JJ, Choi MG, Cho YS, et al. Chronic gastrointestinal symptoms and quality of life in the Korean population. World J Gastroenterol. 2008;14(41):6388-94

42. Lee V, Guthrie E, Robinson A, et al. Functional bowel disorders in primary care: factors associated with health-related quality of life and doctor consultation. J Psychosom Res. 2008;64(2):129-38.

43. Amouretti M, Le PC, Gaudin AF, et al. Impact of irritable bowel syndrome (IBS) on health-related quality of life (HRQOL). Gastroenterol Clin Biol. 2006;30(2):241-46.

44. Pare P, Gray J, Lam S, et al. Health-related quality of life, work productivity, and health care resource utilization of subjects with irritable bowel syndrome: baseline results from LOGIC (Longitudinal Outcomes Study of Gastrointestinal Symptoms in Canada), a naturalistic study. Clin Ther. 2006;28(10):1726-35

45. Coffin B, Dapoigny M, Cloarec D, Comet D, Dyard F. Relationship between severity of symptoms and quality of life in 858 patients with irritable bowel syndrome. Gastroenterol Clin Biol. 2004;28(1):11-15.

46. Le Pen C, Ruszniewski P, Gaudin AF, et al. The burden cost of French patients suffering from irritable bowel syndrome. Scand J Gastroenterol. 2004;39(4):336-43.

47. Si JM, Wang LJ, Chen SJ, Sun LM, Dai N. Irritable bowel syndrome consulters in Zhejiang province: the symptoms pattern, predominant bowel habit subgroups and quality of life. World J Gastroenterol. 2004;10(7):1059-64. 48. Pace F, Molteni P, Bollani S, et al. Inflammatory bowel disease versus irritable bowel syndrome: a hospital-based, case-control study of disease impact on quality of life. Scand J Gastroenterol. 2003;38(10):1031-38. 49. Sandler RS, Everhart JE, Donowitz M, et al. The burden of selected digestive diseases in the United States. Gastroenterology. 2002;122(5):1500-11.

50. Creed F, Ratcliffe J, Fernandez L, et al. Health-related quality of life and health care costs in severe, refractory irritable bowel syndrome. Ann Intern Med. 2001;134(9 Pt 2):860-68.

51. Lee OY, Mayer EA, Schmulson M, Chang L, Naliboff B. Gender-related differences in IBS symptoms. Am J Gastroenterol. 2001;96(7):2184-93.

52. Gralnek IM, Hays RD, Kilbourne A, Naliboff B, Mayer EA. The impact of irritable bowel syndrome on health-related quality of life. Gastroenterology. 2000;119(3):654-60. 